\title{
Hematoma subperiosteal orbitário secundário a barotrauma em aviação civil
}

\author{
Orbital subperiosteal hematoma secondary to barotrauma in civil aviation
}

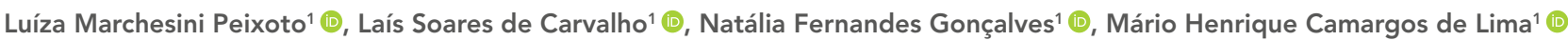

Descritores:

Doenças orbitárias; Diplopia;

Exoftalmia; Hemorragia;

Barotrauma; Aviação; Viagem

$$
\text { aérea }
$$

Keywords:

Orbital diseases; Diplopia;

Exophthalmos; Hemorrhage; Barotrauma; Aviation; Air travel

Recebido: 31/8/2020

Aceito:

10/4/2021

Autor correspondente: Luíza Marchesini Peixoto Avenida Emílio Ribas, 1.819-Gopouva CEP: 07051-000 - Guarulhos, SP, Brasil

E-mail: luizampeixoto@hotmail.com

Instituição: Clínica Oftalmológica Complexo Hospitalar Padre Bento de Guarulhos, SP, Brasil.

Fonte de auxílio à pesquisa: não financiado.

Conflitos de interesse: os autores declaram que não há conflitos de interesses.

\section{RESUMO}

Os diagnósticos diferenciais que compõem as proptoses agudas são, muitas vezes, desafiadores. A anamnese e o exame clínico exigem do oftalmologista atenção especial aos detalhes que permitem diferenciar quadros relativamente benignos e autolimitados de quadros que evoluirão com incapacidades permanentes. Relatamos o caso de uma paciente de 49 anos que, durante viagem de avião, apresentou dor ocular, hematoma periorbitário e proptose do olho esquerdo súbitos. Referia diplopia aguda incapacitante. Exames de tomografia e angiorressonância magnética confirmaram diagnóstico de sinusopatia do seio etmoidal esquerdo e hematoma subperiosteal da órbita esquerda, associado ao barotrauma. Apesar de raro, o diagnóstico de hematoma subperiosteal não traumático deve ser considerado diferencial em relação a proptoses agudas, sendo a anamnese fundamental para essa elucidação diagnóstica.

\section{ABSTRACT}

Differential diagnoses of acute proptosis are often challenging. History and clinical examination require from ophthalmologists special attention to details, which make it possible to differentiate relatively benign and self-limited conditions from those that will progress to permanent disabilities. We report a 49-year-old female patient who had sudden eye pain, periorbital hematoma and proptosis of the left eye during a commercial flight. She also complained of disabling acute diplopia. Computed tomography and magnetic resonance angiography imaging confirmed the diagnosis of subperiosteal hematoma of the left orbit, associated with left ethmoid sinus disease. Although rare, non-traumatic subperiosteal hematoma should be considered in differential diagnoses of acute proptosis, and history taking is fundamental to elucidate the picture. 


\section{INTRODUÇÃO}

As hemorragias orbitárias podem ser classificadas em traumáticas (muito mais comuns) e não traumáticas. Dentre as causas não traumáticas, a segunda causa mais frequente é a hemorragia subperiosteal, ${ }^{(1)}$ apesar de ainda representar entidade clínica infrequente. Geralmente, ela resulta da ruptura de vasos sanguíneos e da formação de hematoma entre o osso e o periósteo separadamente..$^{(2,3)}$

O diagnóstico precoce dos hematomas subperiosteais é essencial para uma intervenção adequada, evitando graves sequelas.

No presente relato, descrevemos o caso de uma paciente que desenvolveu subitamente hematoma subperiosteal secundário a barotrauma associado à sinusopatia crônica identificada em exame de imagem.

Este estudo foi aprovado pelo Comitê de Ética e Pesquisa do Complexo Hospitalar Padre Bento de Guarulhos, em Guarulhos (SP), sob número 3.796.365.

\section{RELATO DE CASO}

Paciente do sexo feminino, 49 anos, foi trazida do Aeroporto Internacional de Guarulhos para o pronto-socorro do nosso serviço com quadro súbito de hematoma periorbitário no olho esquerdo e diplopia vertical, durante voo de Recife a Guarulhos, precedido por cefaleia na região frontal esquerda. Referia tratamento prévio de sinusopatia e negou comorbidades ou coagulopatias prévias.

À ectoscopia, observou-se distopia e proptose no olho esquerdo com hematoma periorbitário (Figura 1). A acuidade visual com melhor correção era 20/20 (1,0) em ambos os olhos. Biomicroscopia, pressão intraocular e fundoscopia não mostravam alterações. Motilidade ocular extrínseca apresentava restrição de abdução, adução, depressão, elevação e exciclodução. Os reflexos fotomotores direto e consensual estavam presentes, sem defeito pupilar aferente relativo.

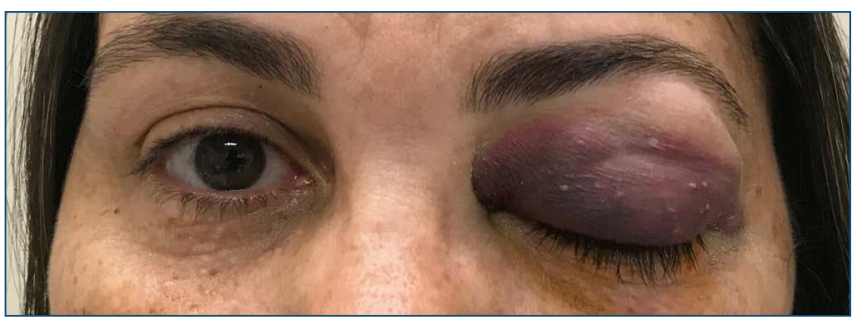

Figura 1. Ectoscopia do primeiro dia.

Solicitamos tomografia computadorizada (TC) de crânio e órbitas com e sem contraste, que mostrou formação amorfa, de provável natureza hemática, obliterando a gordura intraocular na porção medial da órbita esquerda e promovendo discreto desvio lateral dos ventres musculares dos músculos reto superior e oblíquo superior esquerdos (Figura 2).

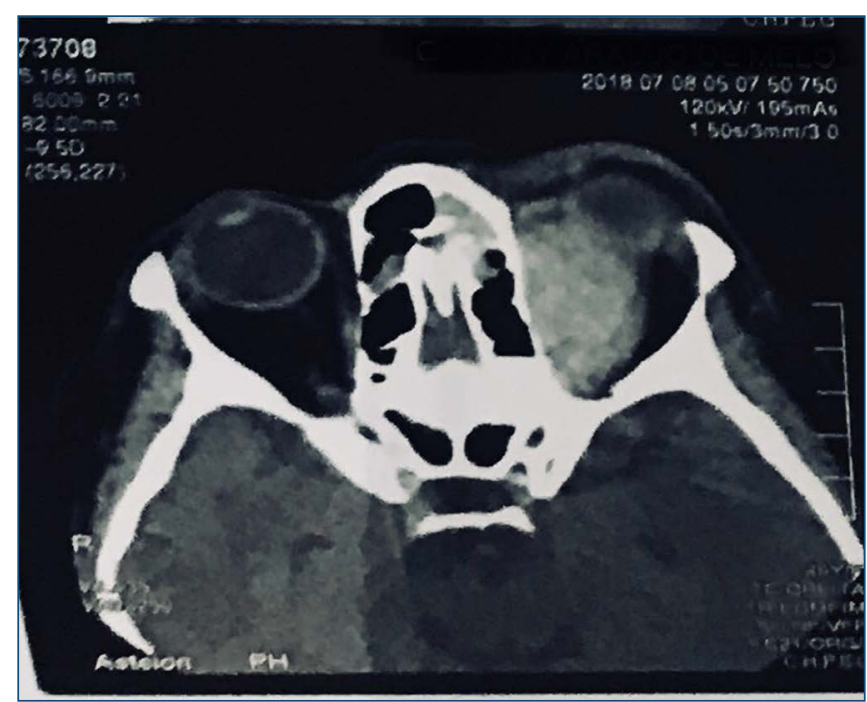

Figura 2. Tomografia computadorizada de órbita sem contraste do primeiro dia.

A ressonância magnética (RM) de crânio e órbitas demonstrou opacificação da luz do seio etmoidal esquerdo, com extensão do processo inflamatório para o compartimento extraconal ipsilateral, com espessamento e deslocamento da musculatura adjacente (Figuras 3 e 4). Exames laboratoriais, como hemograma e coagulograma, não apresentaram anormalidades.

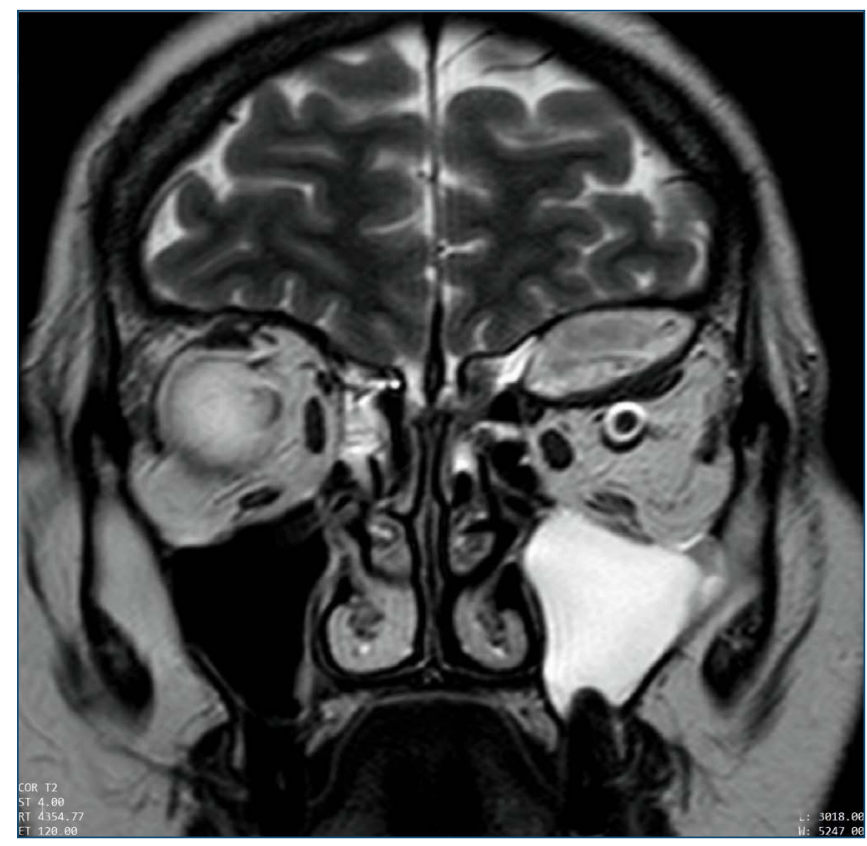

Figura 3. RM no plano coronal, ponderada em T2. 


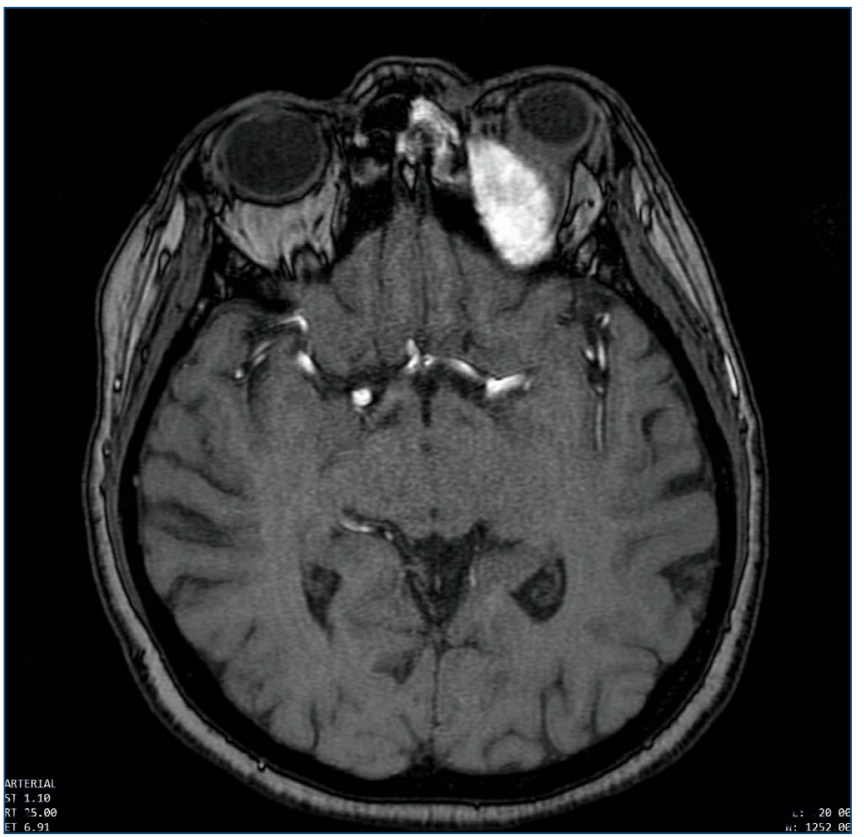

Figura 4. RM no plano axial, ponderada em T1, com contraste.

Foi feita opção por tratamento da sinusopatia com amoxicilina+clavulanato oral e conduta expectante do hematoma, com reavaliações diárias. A angiorressonância magnética (angioRM) de crânio foi realizada ao final do décimo dia de antibioticoterapia, excluiu causas vasculares e corroborou a hipótese diagnóstica de hematoma subperiosteal consequente a um barotrauma associado à sinusopatia crônica (Figura 5). A paciente evoluiu com resolução espontânea do hematoma, assim como remissão completa dos sinais e sintomas apresentados (Figura 6).

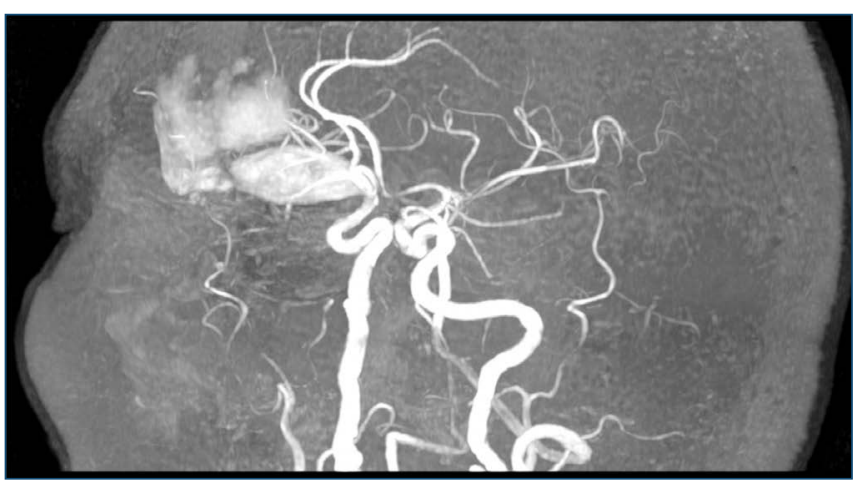

Figura 5. Angiorressonância magnética de crânio em fase arterial.

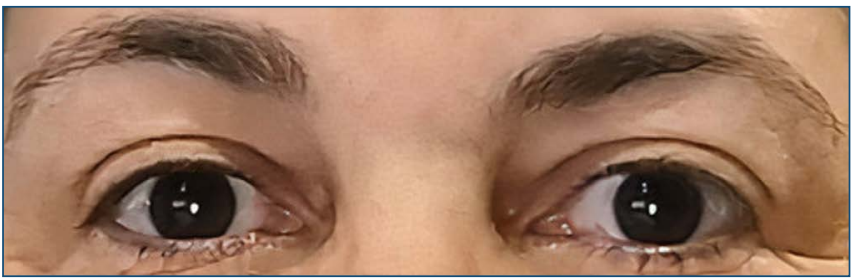

Figura 6. Ectoscopia após 40 dias do quadro inicial.

\section{DISCUSSÃO}

O diagnóstico das lesões hemorrágicas orbitárias torna-se difícil pela raridade e pela forma de apresentação, que geralmente é inespecífica. Essas lesões incluem um grupo heterogêneo de condições idiopáticas ou causadas por traumas, cirurgias, tumores, desordens sanguíneas ou malformações vasculares preexistentes. ${ }^{\left({ }^{1}\right)}$ Anatomicamente, são classificadas em hemorragias intraorbitárias difusas, intraorbitárias localizadas, subperiosteais, relacionadas aos músculos extraoculares e relacionadas a implantes do assoalho da órbita. ${ }^{(4)}$

As hemorragias subperiosteais de órbita são mais frequentes em homens jovens e associadas a trauma, localizando-se principalmente no teto orbitário, na porção do osso frontal, ${ }^{(5)}$ sendo decorrentes de um sangramento localizado no espaço subperiosteal da órbita. ${ }^{(6,7)}$

A fisiopatologia do barotrauma envolve a lei de BoyleMariotte, a qual afirma que, em uma determinada temperatura, o volume de um gás varia inversamente à pressão. Dessa forma, as alterações na pressão barométrica secundárias à mudança na altitude durante o voo podem justificar a etiologia de alterações orbitárias. Durante a decolagem, ocorre redução da pressão ambiente e, de acordo com a lei de Boyle, há expansão gasosa intrassinus contra as paredes externas rígidas dos seios. Em um seio nasal inflamado, com óstios total ou parcialmente obstruídos, a expansão do ar e o aumento da pressão intranasal provocam lesão por compressão da mucosa sinusal contra as paredes externas dos seios ósseos. Na descida do avião, em contrapartida, o aumento da pressão atmosférica provoca redução do volume gasoso na cavidade nasal e seios da face, com consequente descompressão abrupta e efeito de "sucção" ou avulsão dos tecidos, sendo a hemorragia orbitária uma das formas de apresentação clínica. Estudos de aviação e mergulho apontam que o barotrauma está mais relacionado à descompressão (aterrissagem e aumento da gravidade) do que à compressão dos tecidos por expansão volumétrica. ${ }^{(8)}$

De acordo com a cartilha de medicina aeroespacial, condições como sinusite aguda ou crônica constituem contraindicação ao voo. A infecção deve ser tratada com o uso de antibióticos de largo espectro, agentes mucolíticos, descongestionantes orais, corticosteroides e uso temporário de descongestionantes nasais. O uso de solução fisiológica nasal também contribui para a limpeza e a umidificação da mucosa nasal. ${ }^{(9)}$

Sinais e sintomas do hematoma subperiosteal desenvolvem-se subitamente, incluindo proptose, deslocamento do globo ocular, edema da pálpebra superior, dor, 
náuseas e vômitos, diplopia vertical e, em alguns casos, baixa acuidade visual. Apresentações subagudas podem ocorrer, mas são muito menos frequentes. ${ }^{(2)}$ Acuidade visual e função do nervo óptico são os dois fatores primordiais para a decisão de uma intervenção imediata. Além disso, um bom exame de imagem, como a TC com contraste ou a RM, é essencial para a condução do caso. ${ }^{(1)} \mathrm{A}$ TC define nitidamente uma massa não captante de contraste, hiperdensa, biconvexa, com ampla base de implantação, principalmente na porção superior da cavidade orbital (teto da órbita). Já a RM mostra hiperintensidade nas imagens em T2, que, no período crônico, podem se tornar hipointensas, quando o hematoma se liquefaz. ${ }^{(10)}$

Embora a maioria se resolva a longo prazo, algumas hemorragias subperiosteais podem causar sequelas graves, como cegueira, atrofia do nervo óptico, estrabismo secundário, ou até mesmo exoftalmia persistente devido à fibrose e à calcificação do hematoma. (3)

As três opções de manejo dos pacientes são conduta expectante (resolução espontânea), aspiração por agulha e exploração cirúrgica. Aguardar resolução espontânea só é uma opção viável quando não há evidências de risco de lesão do nervo óptico, e a acuidade visual não está comprometida. A drenagem cirúrgica é importante tanto para drenagem do hematoma quanto da sinusite em casos graves, além de viabilizar a confirmação diagnóstica. ${ }^{(11)}$

No caso apresentado, optou-se por conduta expectante, devido à preservação da acuidade visual e à ausência de sinais clínicos ou radiológicos de comprometimento do nervo óptico. Entretanto, a reavaliação diária e o acompanhamento da regressão dos sinais e sintomas foram essenciais para que a conduta conservadora permanecesse como opção viável.

\section{REFERÊNCIAS}

1. McNab AA. Nontraumatic orbital hemorrhage. Surv Ophthalmol. 2014;59(2):166-84.

2. Matsumoto S, Yamamoto T, Ban S, Motozaki T, Sato S, Shingu T, et al. Spontaneous subperiosteal hematoma of the orbit-case report. Neurol Med Chir (Tokyo). 1994;34(1):27-9.

3. Chaves JBL, Batista MSA, Piske RL, Cunha KA, Almeida KJ. Hematoma subperiosteal de órbita: relato de caso. Arq Bras Oftalmol. 2007;70(4):693-7.

4. Mete A, Pamukcu C, Mete A, Kimyon S, Mete D, Gözen I, Saygili O. Intraorbital haematoma during a commercial flight: a case report. BMC Ophthalmol. 2015;15:47.

5. Park HW, Lee BJ, Chung YS. Orbital subperiosteal hematoma associated with sinus infection. Rhinology. 2010;48(1):117-22.

6. Atalla ML, McNab AA, Sullivan TJ, Sloan B. Nontraumatic subperiosteal orbital hemorrhage. Ophthalmology. 2001;108(1):183-9.

7. Crawford C, Mazzoli R. Subperiosteal hematoma in multiple settings. Digit J Ophthalmol. 2013;19(1):6-8.

8. Vaezeafshar R, Psaltis AJ, Rao VK, Zarabanda D, Patel ZM, Nayak JV. Barosinusitis: Comprehensive review and proposed new classification system. Allergy Rhinol (Providence). 2017;8(3):109-17.

9. Faculdade de Ciências Médicas da Santa Casa de São Paulo. Conselho Federal de Medicina. Doutor, posso viajar de avião? Cartilha de Medicina Aeroespacial. Brasília, DF: Conselho Federal de Medicina; 2011 [citado 2021 Mar 23]. 72 p. Disponível em: https://portal.cfm.org.br/images/ stories/pdf/cartilha_medicina_aeroespacialfinal2.pdf

10. Meltzer DE, Mirbagheri S, Aygun N. Subperiosteal hematoma of the orbit: a variety of presentations. J Radiol Case Rep. 2019;13(6):13-23.

11. Sabet SJ, Tarbet KJ, Lemke BN, Smith ME, Albert DM. Subperiosteal hematoma of the orbit with osteoneogenesis. Arch Ophthalmol. 2001;119(2):301-3. 